\title{
Efeitos de genótipos, ambientes e de tratamentos hidrotérmicos na concentração de isoflavonas agliconas em grãos de soja ${ }^{(1)}$
}

\author{
Mercedes Concórdia Carrão-Panizzi(2), Aline Silva Simão(3) e Akio Kikuchi(4)
}

\begin{abstract}
Resumo - O objetivo deste trabalho foi avaliar os efeitos de tratamentos hidrotérmicos, da variabilidade genética e de ambientes no teor de isoflavonas agliconas em grãos de soja [Glycine max (L.) Merrill]. O estudo foi realizado com as cultivares de soja BR 36, FEPAGRO RS-10 e BRS 155, cultivadas em Londrina, em Capanema e em Palmas, PR, na safra 1999/2000, mediante tratamentos hidrotérmicos de 40,50 e $60^{\circ} \mathrm{C}$ por 12 e 18 horas. As maiores concentrações de isoflavonas totais $(280 \mathrm{mg} / 100 \mathrm{~g}$ ) foram observadas nos grãos colhidos em Palmas, onde a temperatura média durante o enchimento de vagens foi mais baixa $\left(19^{\circ} \mathrm{C}\right)$. Em Londrina $\left(23^{\circ} \mathrm{C}\right)$, houve menor concentração de isoflavonas $(140 \mathrm{mg} / 100 \mathrm{~g})$. A cultivar BRS 155 apresentou o maior teor de isoflavonas totais em Palmas e em Londrina. Grãos nãotratados da BRS 155 apresentaram em média $4,0 \mathrm{mg} / 100 \mathrm{~g}$ de agliconas, as quais aumentaram para $52 \mathrm{mg} / 100 \mathrm{~g}$, após os tratamentos hidrotérmicos. $\mathrm{O}$ tratamento a $50^{\circ} \mathrm{C}$ por 12 horas foi mais efetivo no desenvolvimento de isoflavonas agliconas. $\mathrm{A} 60^{\circ} \mathrm{C}$, houve redução das agliconas, decorrente da inativação das $\beta$-glicosidases. As formas malonil, que são termicamente instáveis, também foram reduzidas nas temperaturas mais altas.
\end{abstract}

Termos para indexação: Glycine max, glicosídeo, alimento funcional, tratamento térmico.

\section{Effects of genotypes, environments and hydrothermal treatments on the isoflavone aglycone concentration in soybean grains}

\begin{abstract}
The objective of this work was to assess the effects of hydrothermal treatments and the environmental and genetic variability on the isoflavone aglycones concentration in soybean [Glycine $\max ($ L.) Merrill] grains. The study was carried out with the soybean cultivars BR 36, FEPAGRO RS-10, and BRS 155, cultivated in three locals of Paraná State (Londrina, Capanema, and Palmas), during 1999/ 2000 soybean season, submitted to hydrothermal treatments at 40,50 and $60^{\circ} \mathrm{C}$ for 12 and 18 hours. Higher concentrations of total isoflavones $(280 \mathrm{mg} / 100 \mathrm{~g}$ ) were observed in grains harvested in Palmas, where the average temperature during pod filling was $19^{\circ} \mathrm{C}$. In Londrina $\left(23^{\circ} \mathrm{C}\right)$, there was lower concentration of isoflavones $(140 \mathrm{mg} / 100 \mathrm{~g})$. The cultivar BRS 155 presented the highest content of total isoflavones in Palmas and in Londrina. Non-treated grains of BRS 155 showed in average $4.0 \mathrm{mg} / 100 \mathrm{~g}$ of aglycones, which increased to $52 \mathrm{mg} / 100 \mathrm{~g}$, after hydrothermal treatments. The treatment at $50^{\circ} \mathrm{C}$ for 12 hours was the most effective in developing isoflavone aglycones. At $60^{\circ} \mathrm{C}$, there was a decrease of the aglycones, due to inactivation of $\beta$-glycosidases. Malonyl forms, which are thermal unstable, were reduced at higher temperatures.
\end{abstract}

Index terms: Glycine max, glycosides, functional foods, heat treatment.

(1)Aceito para publicação em 29 de maio de 2003.

(2) Embrapa-Labex/USDA-ARS, National Center for Agricultural Utilization Research (NCAUR), $1815 \mathrm{~N}$ University St., Peoria, IL, 61604, USA. E-mail: panizzim@ncaur.usda.gov

(3) Embrapa-Centro Nacional de Pesquisa de Soja, Caixa Postal 231, CEP 86001-970 Londrina, PR. E-mail aline.quimica@bol.com.br

(4) National Agricultural Research Center for Western Region, Department of Crop Breeding, Soybean Breeding Laboratory, Kagawa 765-8508, Japão. E-mail: akiokiku@affrc.go.jp

\section{Introdução}

No Brasil, a soja tem recebido atenção pública considerável, por seus potenciais benefícios para a saúde humana, prevenindo doenças crônicas. Estudos epidemiológicos mostraram uma incidência menor de câncer relacionado com hormônios na Ásia do que em países Ocidentais. A genisteína é o principal fator na prevenção do câncer (Peterson \& Barnes, 1993). A dieta tradicional dos países asiáticos, rica em soja e com altos teores de 
isoflavonas, foi fator determinante em estudos conduzidos sobre este tema (Coward et al., 1993).

As isoflavonas ocorrem naturalmente nos grãos de soja e na maioria dos produtos de soja ocidentais, como $\beta$-glicosídeos conjugados (Coward et al., 1998). Beta-glicosidases (Matsuura et al., 1989) e enzimas intestinais (Tam et al., 1998) hidrolisam as isoflavonas conjugadas glicosídicas desenvolvendo as formas agliconas. Vários estudos estão sendo realizados para definir como ocorre a absorção intestinal destes compostos em humanos. Setchell et al. (2001) constataram que as isoflavonas glicosídicas não são absorvidas intactas: a biodisponibilidade requer uma hidrólise inicial do açúcar, para que sejam transportadas pela circulação periférica.

As isoflavonas agliconas (daidzeína e genisteína), compostos mais prontamente disponíveis, são encontradas em concentrações maiores em alimentos como "tempeh" e "miso". O processo de fermentação na obtenção destes alimentos causa redução dos glicosídeos e formação de agliconas (Coward et al., 1998). Variabilidade genética e efeitos do ambiente afetam as concentrações de isoflavonas em grãos de soja (Tsukamoto et al., 1995; Carrão-Panizzi et al., 1998, 1999). Métodos de processamento associados a matéria-prima com altos níveis de isoflavonas podem favorecer maior desenvolvimento de isoflavonas agliconas.

O objetivo deste trabalho foi avaliar os efeitos de tratamentos hidrotérmicos, variabilidade genética das cultivares de soja, e diferentes locais de cultivo, no teor de isoflavonas agliconas.

\section{Material e Métodos}

Foram realizados dois experimentos, usando-se o delineamento inteiramente casualizado em esquema fatorial com três repetições. No primeiro experimento, os tratamentos foram constituídos pela combinação de três cultivares de soja (BRS 155, FEPAGRO RS-10 e BR 36), três locais de cultivo (safra 1999/2000) no Estado do Paraná (Palmas, Londrina e Capanema) e dois tratamentos hidrotérmicos dos grãos de soja imersos por 18 horas, em água destilada e deionizada, sob temperaturas de 50 e $60^{\circ} \mathrm{C}$. No segundo experimento, os tratamentos foram constituídos pela combinação de três locais de cultivo (Palmas, Londrina e Capanema), três temperaturas (40, 50 e $60^{\circ} \mathrm{C}$ ), e dois períodos de imersão (12 e 18 horas) dos grãos da cultivar de soja BRS 155 em água destilada e deionizada. Após os tratamentos hidrotérmicos, os grãos (amostras de $10 \mathrm{~g}$ ) foram lavados com água fria, secados em estufa ventilada e moídos em moinhos domésticos (Braun model KSM 2B).

$\mathrm{Na}$ extração das isoflavonas, amostras de $100 \mathrm{mg}$ de grãos de soja moídos foram colocadas em tubos de ensaio com $4,0 \mathrm{~mL}$ de etanol aquoso a $70 \%$, contendo $0,1 \%$ de ácido acético, à temperatura ambiente por cinco horas, e agitados a cada 15 minutos.

Nas análises de cromatografia líquida de alta eficiência (CLAE), 1,5 mL de amostra foi centrifugado por 10 minutos a $13.500 \mathrm{rpm}$, a $10^{\circ} \mathrm{C}$ (Centrífuga Eppendorf mod. 5417R). A seguir, $80 \mu \mathrm{L}$ do sobrenadante foram transferidos para as bandejas do auto-aplicador de amostras do cromatógrafo e $10 \mu \mathrm{L}$ foram injetados no cromatógrafo. As análises de isoflavonas foram realizadas em colunas de cromatografia ODS C-18 (YMC-Pack ODS-AM), S-5 mm, 120 A (250 x 4,6 mm I.D.). Na fase móvel, o solvente A foi acetonitrila com $0,1 \%$ de ácido acético e o solvente B foi água com $0,1 \%$ de ácido acético. As condições iniciais foram $20 \%$ do solvente B em gradiente linear, passando para $50 \%$, depois de 20 minutos. O efluente foi monitorado a $260 \mathrm{~nm}$. As soluções padrões de daidzina, daidzeína, genistina e genisteína foram preparadas de padrões da SIGMA.

Antes de testar os tratamentos pela ANOVA, os dados foram avaliados quanto à distribuição normal (Shapiro \& Wilk, 1965), homogeneidade de variância (Hartley, 1940; Burr \& Foster, 1972) e aditividade do modelo (Tukey, 1949). As médias foram comparadas pelo teste de Tukey a 5\% de probabilidade (Cochran \& Cox, 1957). As análises estatísticas foram realizadas por meio do sistema SAS Institute (1997).

\section{Resultados e Discussão}

Em Palmas, onde a temperatura média durante o período de enchimento de grãos foi de $19,2^{\circ} \mathrm{C}$, houve maior desenvolvimento de isoflavonas (Figura 1). Em Londrina e Capanema, as temperaturas médias mais altas de $23,2^{\circ} \mathrm{C}$ e de $23,7^{\circ} \mathrm{C}$, respectivamente, podem ter sido responsáveis pela redução no teor de isoflavonas. Temperaturas mais baixas durante o enchimento de vagens são determinantes no aumento da concentração de isoflavonas em soja (Tsukamoto et al., 1995), assim como os locais de cultivo (Eldridge \& Kwolek, 1983; Kitamura et al., 1991).

No Brasil, a soja é produzida em regiões de latitudes de $33^{\circ} \mathrm{S}$ até o Equador, e os efeitos dessas amplas condições ambientais, na concentração de isoflavonas, foram registrados por Carrão-Panizzi et al. (1999). Apesar de Palmas e Capanema estarem localizadas quase na mesma latitude $\left(26^{\circ} \mathrm{S}\right.$ e $\left.25^{\circ} \mathrm{S}\right)$, 
as diferenças nas altitudes ( $1.035 \mathrm{~m}$ e $368 \mathrm{~m}$ ) causam diferenças ambientais responsáveis pelas concentrações diferentes de isoflavonas. Quando os grãos foram tratados hidrotermicamente, ocorreu $40 \%$ de redução no teor de isoflavonas, mas as diferenças observadas entre os locais foram mantidas (Figura 1A).

Em grãos secos de soja sob boas condições de armazenamento não há formação de agliconas. Neste estudo, os grãos que não sofreram tratamento hidrotérmico apresentaram concentrações totais das agliconas muito reduzidas (Figura 1A). Após os tratamentos térmicos, as concentrações de agliconas nos grãos aumentaram oito vezes, enquanto as diferenças ambientais permaneceram. Quando

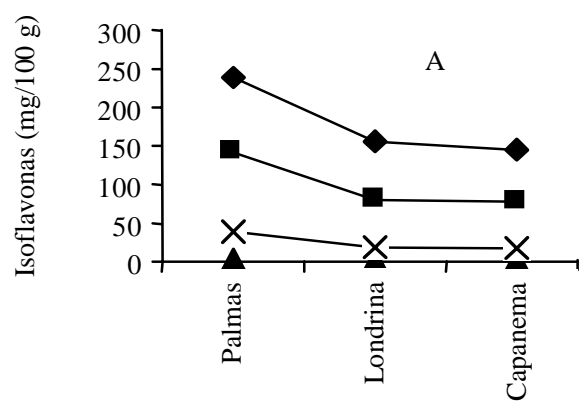

Locais

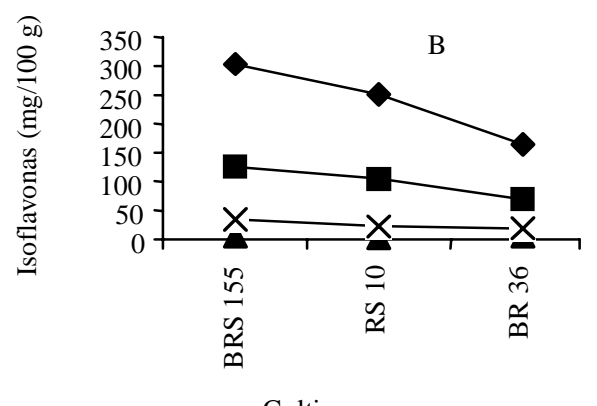

Cultivares

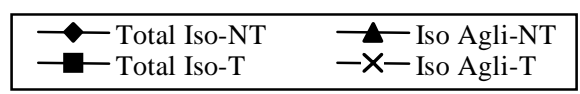

Figura 1. Valor médio das concentrações de isoflavonas totais (Total Iso) e de isoflavonas agliconas (Iso Agli) em grãos tratados (T) e não-tratados (NT) hidrotermicamente por 18 horas a $50^{\circ} \mathrm{C}$ e $60^{\circ} \mathrm{C}$, de três cultivares de soja semeadas em diferentes locais do Estado do Paraná(A), e de cultivares de soja semeadas em Palmas, no Estado do Paraná (B). semeada em Palmas, a cultivar BRS 155 apresentou os níveis mais altos de isoflavonas totais em grãos tratados e não-tratados (Figura 1B), o que pode ser um efeito genético. Essa variabilidade também foi observada quando houve o desenvolvimento de agliconas. ABRS 155 é uma cultivar de soja especial, que apresenta um terço da concentração de inibidor de tripsina normalmente registrada em cultivares convencionais (Carrão-Panizzi et al., 2000). A cultivar BR 36 tem sido reportada como uma cultivar que apresenta reduzido teor de isoflavona (Carrão-Panizzi \& Kitamura, 1995; Carrão-Panizzi et al., 1998). No presente estudo, essa cultivar também apresentou os menores teores de isoflavonas em todos os locais de cultivo. No Brasil, a 'BR 36' é muito cultivada pelos produtores de soja orgânica, por causa do seu sabor mais suave, do tamanho moderado dos grãos e da cor do hilo (marrom-claro) que aumentam sua aceitabilidade para o consumo humano. Entretanto, o teor reduzido de isoflavonas pode dificultar seu uso no processamento de alimentos funcionais.

A $50^{\circ} \mathrm{C}$, houve um maior desenvolvimento de isoflavonas agliconas (Tabela 1 ). A $60^{\circ} \mathrm{C}$, a redução no teor de agliconas pode ter sido decorrente da inativação das $\beta$-glicosidases, como observado por Matsuura \& Obata (1993). Em Palmas, a cultivar BRS 155 apresentou maiores concentrações daqueles compostos do que as cultivares BRS 36 e RS-10. O local de cultivo afetou as concentrações totais de isoflavonas do mesmo modo que afetou o total de agliconas (Tabela 1). As concentrações totais de isoflavonas diminuíram após tratamentos hidrotérmicos dos grãos por 18 horas (Tabela 2).

Tabela 1. Concentração de agliconas e isoflavonas totais $(\mathrm{mg} / 100 \mathrm{~g})$ em grãos de cultivares de soja semeadas em diferentes locais do Estado do Paraná, submetidos a tratamentos hidrotérmicos por 18 horas $^{(1)}$.

\begin{tabular}{|c|c|c|c|c|c|c|}
\hline \multirow[t]{2}{*}{ Cultivares } & \multicolumn{2}{|c|}{ Palmas } & \multicolumn{2}{|c|}{ Londrina } & \multicolumn{2}{|c|}{ Capanema } \\
\hline & $50^{\circ} \mathrm{C}$ & $60^{\circ} \mathrm{C}$ & $50^{\circ} \mathrm{C}$ & $60^{\circ} \mathrm{C}$ & $50^{\circ} \mathrm{C}$ & $60^{\circ} \mathrm{C}$ \\
\hline \multicolumn{7}{|c|}{ Agliconas } \\
\hline 3R 36 & $37,6 \mathrm{bA} a$ & $25,1 \mathrm{bB} a$ & $19,4 \mathrm{bA} b$ & $7,1 \mathrm{aB} b$ & $17,5 \mathrm{bA} b$ & $7,2 \mathrm{bB} b$ \\
\hline-10 & $41,8 \mathrm{bA} a$ & $20,0 \mathrm{bB} a$ & $28,8 \mathrm{aA} b$ & $9,0 \mathrm{aB} b$ & $24,5 \mathrm{abA} b$ & $10,9 \mathrm{abB} b$ \\
\hline RS 155 & $75,7 \mathrm{aA} a$ & $34,6 \mathrm{aB} a$ & $36,7 \mathrm{aA} b$ & $12,8 \mathrm{aB} b$ & $32,3 \mathrm{aA} b$ & $17,9 \mathrm{aB} b$ \\
\hline \multicolumn{7}{|c|}{ Isoflavonas totais } \\
\hline BR 36 & $105,9 \mathrm{cA} a$ & $90,6 \mathrm{cB} a$ & $70,2 \mathrm{bA} b$ & $54,1 \mathrm{bB} b$ & $53,6 \mathrm{cA} b$ & $46,0 \mathrm{cA} b$ \\
\hline RS-10 & $153,1 \mathrm{bA} a$ & $125,8 \mathrm{bB} a$ & $93,9 \mathrm{aA} b$ & $93,9 \mathrm{aA} b$ & $81,8 \mathrm{bA} b$ & $78,7 \mathrm{bA} b$ \\
\hline BRS 155 & $195,7 \mathrm{aA} a$ & $180,6 \mathrm{aB} a$ & $88,7 \mathrm{aA} c$ & $775 \mathrm{~s}$ & $108,0 \mathrm{aA} b$ & $102,7 \mathrm{aA} b$ \\
\hline \multicolumn{7}{|c|}{$\begin{array}{l}\text { (1) Médias seguidas pela mesma letra minúscula nas colunas não diferem } \\
\text { entre si pelo teste de Tukey, a 5\% de probabilidade; médias seguidas pela } \\
\text { mesma letra maiúscula nas linhas, em cada local, não diferem entre si pelo } \\
\text { teste de Tukey, a 5\% de probabilidade; médias seguidas pela mesma letra } \\
\text { em itálico nas linhas, em cada temperatura, não diferem entre si pelo teste } \\
\text { de Tukey, a 5\% de probabilidade. }\end{array}$} \\
\hline
\end{tabular}


Tabela 2. Concentração dos compostos isoflavonas (mg/100 g), em grãos de cultivares de soja produzidos em Palmas, no Estado do Paraná, submetidos a tratamentos hidrotérmicos por 18 horas $^{(1)}$.

\begin{tabular}{lccccccc}
\hline Tratamento & Daidzina & Genistina & Malonil daidzina & Malonil genistina & Daidzeína & Genisteína & Isoflavona total \\
\hline & & & & & & & \\
Grãos não-tratados & $20,6 \mathrm{a}$ & $28,7 \mathrm{c}$ & $78,5 \mathrm{a}$ & $120,7 \mathrm{a}$ & $0,8 \mathrm{c}$ & $1,2 \mathrm{c}$ & $250,5 \mathrm{a}$ \\
$50^{\circ} \mathrm{C}$ & $21,9 \mathrm{a}$ & $32,9 \mathrm{~b}$ & $16,7 \mathrm{~b}$ & $39,8 \mathrm{~b}$ & $18,1 \mathrm{a}$ & $23,7 \mathrm{a}$ & $153,1 \mathrm{~b}$ \\
$60^{\circ} \mathrm{C}$ & $25,0 \mathrm{a}$ & $42,4 \mathrm{a}$ & $12,2 \mathrm{~b}$ & $26,1 \mathrm{c}$ & $8,6 \mathrm{~b}$ & $11,1 \mathrm{~b}$ & $125,8 \mathrm{c}$ \\
\hline & & & & & & & \\
Grãos não-tratados & $11,0 \mathrm{a}$ & $19,2 \mathrm{~b}$ & $45,5 \mathrm{a}$ & $84,4 \mathrm{a}$ & $1,6 \mathrm{c}$ & $2,6 \mathrm{c}$ & $164,5 \mathrm{a}$ \\
$50^{\circ} \mathrm{C}$ & $8,9 \mathrm{a}$ & $19,4 \mathrm{~b}$ & $10,2 \mathrm{~b}$ & $30,0 \mathrm{~b}$ & $15,5 \mathrm{a}$ & $22,1 \mathrm{a}$ & $106,0 \mathrm{~b}$ \\
$60^{\circ} \mathrm{C}$ & $11,2 \mathrm{a}$ & $26,0 \mathrm{a}$ & $7,3 \mathrm{c}$ & $19,8 \mathrm{c}$ & $10,7 \mathrm{~b}$ & $14,4 \mathrm{~b}$ & $90,6 \mathrm{bc}$ \\
\hline & & & & BRS 155 & & & \\
Grãos não-tratados & $23,4 \mathrm{a}$ & $35,3 \mathrm{a}$ & $96,1 \mathrm{a}$ & $143,8 \mathrm{a}$ & $1,6 \mathrm{c}$ & $2,3 \mathrm{c}$ & $302,5 \mathrm{a}$ \\
$50^{\circ} \mathrm{C}$ & $15,6 \mathrm{~b}$ & $26,2 \mathrm{~b}$ & $15,2 \mathrm{~b}$ & $28,2 \mathrm{~b}$ & $21,0 \mathrm{a}$ & $29,8 \mathrm{a}$ & $195,7 \mathrm{~b}$ \\
$60^{\circ} \mathrm{C}$ & $26,2 \mathrm{a}$ & $39,2 \mathrm{a}$ & $12,7 \mathrm{~b}$ & $22,8 \mathrm{bc}$ & $11,3 \mathrm{~b}$ & $15,2 \mathrm{~b}$ & $180,6 \mathrm{bc}$ \\
\hline
\end{tabular}

${ }^{(1)}$ Médias seguidas pela mesma letra nas colunas não diferem entre si pelo teste de Tukey, a $5 \%$ de probabilidade.

A $60^{\circ} \mathrm{C}$, as isoflavonas glicosídicas, daidzina e genistina aumentaram suas concentrações. Comparando-se os grãos não-tratados com os que receberam tratamentos hidrotérmicos, as formas malonil foram acentuadamente reduzidas. As formas malonil são termicamente instáveis e sob altas temperaturas $\left(80^{\circ} \mathrm{C}\right)$ se convertem nas formas glicosídicas (Kudou et al., 1991). A 50 ${ }^{\circ} \mathrm{C}$, houve maior desenvolvimento de isoflavonas agliconas (Tabela 2).

As concentrações e as formas de isoflavonas variam nos produtos de soja em decorrência de diferentes métodos de processamento (Coward et al., 1998). Para que ocorra absorção após administração oral, as isoflavonas glicosídicas dos grãos de soja requerem uma hidrólise inicial do açúcar para serem convertidas em daidzeína e genisteína, que são as formas biologicamente ativas (Setchell et al., 2001). Isoflavonas glicosídicas são hidrolisadas pelas enzimas $\beta$-glicosidases, que têm atividade ótima em $\mathrm{pH}$ entre 4,3 e 7,0 e à temperatura de $45^{\circ} \mathrm{C}$ (Matsuura \& Obata, 1993). A hidrólise é tempo-dependente e as $\beta$-glicosidases gradualmente perdem atividade. A $55^{\circ} \mathrm{C}, 80 \%$ da atividade original foi mantida, enquanto a $60^{\circ} \mathrm{C}$, a enzima foi inativada (Matsuura \& Obata, 1993). Park et al. (2001) verificaram que a enzima $\beta$-glicosidase de Aspergillus oryzae foi mais eficaz na hidrólise das formas glicosídicas do que nas formas malonil glicosídicas.

Os efeitos dos tratamentos hidrotérmicos foram evidenciados no experimento que considerou somente a cultivar BRS 155, semeada em Palmas. $\mathrm{O}$ tratamento hidrotérmico a $50^{\circ} \mathrm{C}$ por 12 horas proporcionou melhor condição para o desenvolvimento de isoflavonas agliconas, daidzeína e genisteína (Tabela 3). O tratamento de 18 horas a
Tabela 3. Concentração de agliconas e de isoflavonas totais (mg/100 g) em grãos de soja da cultivar BRS 155, semeada em diferentes locais do Estado do Paraná, submetidos a tratamentos hidrotérmicos por períodos de tempos definidos ${ }^{(1)}$.

\begin{tabular}{|c|c|c|c|c|c|c|}
\hline \multirow{2}{*}{$\begin{array}{l}\text { Tempe- } \\
\text { ratura }\end{array}$} & \multicolumn{2}{|c|}{ Palmas } & \multicolumn{2}{|c|}{ Londrina } & \multicolumn{2}{|c|}{ Capanema } \\
\hline & 12 horas & 18 horas & 12 horas & 18 horas & 12 horas & 18 horas \\
\hline & \multicolumn{6}{|c|}{ Agliconas } \\
\hline $40^{\circ} \mathrm{C}$ & $67,9 \mathrm{bB} a$ & $87,4 \mathrm{aA} a$ & $24,3 \mathrm{aB} b$ & $35,7 \mathrm{aA} b$ & $26,1 \mathrm{bB} b$ & $46,5 \mathrm{a} A b$ \\
\hline $50^{\circ} \mathrm{C}$ & $86,3 \mathrm{aA} a$ & $75,7 \mathrm{aA} a$ & $41,0 \mathrm{aA} b$ & $36,7 \mathrm{aA} b$ & $44,9 \mathrm{aA} b$ & $32,3 \mathrm{aAB} b$ \\
\hline $60^{\circ} \mathrm{C}$ & $27,2 \mathrm{aC} a$ & $34,6 \mathrm{aB} a$ & $13,7 \mathrm{aB} a$ & $12,8 \mathrm{aB} b$ & $17,9 \mathrm{aB} a$ & $17,9 \mathrm{aB} b$ \\
\hline \multicolumn{7}{|c|}{ Isoflavonas totais } \\
\hline $40^{\circ} \mathrm{C}$ & $274,0 \mathrm{aA} a$ & $241,4 \mathrm{bA} a$ & $126,8 \mathrm{aA} b$ & $133,2 \mathrm{aA} b$ & $151,4 \mathrm{aA} b$ & $143,0 \mathrm{aA} b$ \\
\hline $50^{\circ} \mathrm{C}$ & $225,4 \mathrm{aB} a$ & $195,7 \mathrm{bB} a$ & $159,0 \mathrm{aA} b$ & $88,7 \mathrm{bB} b$ & $120,8 \mathrm{aAB} c$ & $107,0 \mathrm{aB} b$ \\
\hline $60^{\circ} \mathrm{C}$ & $167,1 \mathrm{aC} a$ & $180,6 \mathrm{aB} a$ & $78,3 \mathrm{aB} b$ & $77,5 \mathrm{aB} b$ & $101,9 \mathrm{aB} b$ & $102,7 \mathrm{aB} b$ \\
\hline
\end{tabular}
não diferem entre si pelo teste de Tukey, a 5\% de probabilidade; médias seguidas pela mesma letra maiúscula nas colunas não diferem entre si pelo teste de Tukey, a 5\% de probabilidade; médias seguidas pela mesma letra em itálico nas linhas, em cada período de tempo, não diferem entre si pelo teste de Tukey, a 5\% de probabilidade.

$40^{\circ} \mathrm{C}$ foi similar ao anterior. Nesse caso, a temperatura menor foi compensada pelo período de exposição mais longo. A $60^{\circ} \mathrm{C}$, as concentrações de agliconas diminuíram em ambos os períodos. A exposição prolongada à temperatura mais alta reduziu os teores totais de isoflavonas (Tabela 3). Os resultados desse segundo experimento seguiram a mesma tendência observada no primeiro, ou seja, quando a temperatura foi mais alta e o período de exposição foi mais longo, as formas malonil reduziram, ao passo que os glicosídeos, daidzina e genistina aumentaram (Tabela 4). Coward et al. (1998) observaram que a extração aquosa a quente aumenta o teor dos glicosídeos. Maiores concentrações de daidzeína e genisteína foram observadas nos tratamentos a $50^{\circ} \mathrm{C}$ por 12 horas e $40^{\circ} \mathrm{C}$ por 18 horas (Tabela 4 ). 
Tabela 4. Concentrações dos compostos de isoflavonas ( $\mathrm{mg} / 100 \mathrm{~g}$ ) em grãos da cultivar de soja BRS 155 semeada em diferentes locais do Estado do Paraná, em razão da temperatura e do tempo de submersão (12 e 18 horas) em água destilada e deionizada ${ }^{(1)}$.

\begin{tabular}{|c|c|c|c|c|c|c|c|c|c|c|c|c|}
\hline \multirow{2}{*}{$\begin{array}{l}\text { Tempe } \\
\text {-ratura }\end{array}$} & \multicolumn{2}{|c|}{ Daidzina } & \multicolumn{2}{|c|}{ Genistina } & \multicolumn{2}{|c|}{ Malonil daidzina } & \multicolumn{2}{|c|}{ Malonil genistina } & \multicolumn{2}{|c|}{ Daidzeína } & \multicolumn{2}{|c|}{ Genisteína } \\
\hline & 12 & 18 & 12 & 18 & 12 & 18 & 12 & 18 & 12 & 18 & 12 & 18 \\
\hline $40^{\circ} \mathrm{C}$ & $27,9 \mathrm{aA}$ & $12,1 \mathrm{cB}$ & $30,7 \mathrm{bA}$ & $15,6 \mathrm{cB}$ & $58,0 \mathrm{aA}$ & $47,7 \mathrm{aB}$ & $89,5 \mathrm{aA}$ & $78,4 \mathrm{aB}$ & $27,6 \mathrm{bB}$ & $35,1 \mathrm{aA}$ & $40,3 \mathrm{aB}$ & $52,3 \mathrm{aA}$ \\
\hline $50^{\circ} \mathrm{C}$ & $14,8 \mathrm{bB}$ & $22,6 \mathrm{bA}$ & $25,8 \mathrm{bB}$ & $36,0 \mathrm{bA}$ & $37,1 \mathrm{bA}$ & $18,8 \mathrm{bB}$ & $61,4 \mathrm{bA}$ & $42,5 \mathrm{bB}$ & $36,8 \mathrm{aA}$ & $32,4 \mathrm{aA}$ & $49,4 \mathrm{aA}$ & $43,3 \mathrm{aA}$ \\
\hline $60^{\circ} \mathrm{C}$ & $31,4 \mathrm{aA}$ & $34,6 \mathrm{aA}$ & $47,1 \mathrm{aB}$ & $56,1 \mathrm{aA}$ & $20,6 \mathrm{cA}$ & $18,7 \mathrm{bA}$ & $40,7 \mathrm{cA}$ & $36,7 \mathrm{bA}$ & $11,9 \mathrm{cA}$ & $14,3 \mathrm{bA}$ & $15,3 \mathrm{bA}$ & $20,2 \mathrm{bA}$ \\
\hline $\mathrm{CV}(\%)$ & \multicolumn{2}{|c|}{16,1} & \multicolumn{2}{|c|}{13,2} & \multicolumn{2}{|c|}{18,7} & \multicolumn{2}{|c|}{12,9} & \multicolumn{2}{|c|}{15,1} & \multicolumn{2}{|c|}{15,9} \\
\hline
\end{tabular}

(1)Médias seguidas pela mesma letra minúscula nas colunas, e pela mesma letra maiúscula nas linhas, em cada composto, não diferem entre si pelo teste de Tukey, a $5 \%$ de probabilidade.

A escolha de cultivares, que geneticamente apresentam teores elevados de isoflavonas, de locais de cultivo, onde a temperatura média é mais baixa durante o período de enchimento de grãos, e de tratamentos hidrotérmicos dos grãos, com temperaturas e períodos de tempo adequados, proporciona maior desenvolvimento de isoflavonas agliconas (compostos biodisponíveis responsáveis por efeitos benéficos à saúde humana), garantindo a obtenção de matéria-prima mais adequada para processamentos de alimentos funcionais à base de soja.

\section{Conclusões}

1. A variabilidade no teor de isoflavonas na soja é determinada geneticamente.

2. Temperaturas mais frias durante o período de enchimento de vagens aumentam as concentrações de isoflavonas.

3. O tratamento hidrotérmico dos grãos a $50^{\circ} \mathrm{C}$ por 12 horas é eficiente no desenvolvimento de isoflavona agliconas.

\section{Agradecimentos}

Aos Drs. José Marcos Gontijo Mandarino e José Erivaldo, da Embrapa-Centro Nacional de Pesquisa de Soja, pelo suporte nas análises químicas e pelo auxílio na análise estatística.

\section{Referências}

BURR, I. W.; FOSTER, L. A. A test for equality of variances. West Lafayette: University of Purdue, 1972. 26 p. (Mimeo Series, 282).
CARRÃO-PANIZZI, M. C.; ALMEIDA, L. A.; KIIHL, R. A. S.; MIRANDA, L. C.; KIKUCHI, A.; MANDARINO, J. M. G.; BORDIGNON, J. R.; SHIMANUKI, S.; DEGAWA, H.; TSUKAMOTO, C. Breeding efforts for nutritional and food processing quality of soybean at Embrapa, Brazil. In: INTERNATIONAL SOYBEAN PROCESSING AND UTILIZATION CONFERENCE, 3., 2000, Tsukuba. Proceedings... Tsukuba: The Japanese Society for Food Science and Technology, 2000. p. 37-40.

CARRÃO-PANIZZI, M. C.; BelÉIA， A. D. P.; KITAMURA, K.; OLIVEIRA, M. C. N. Effects of genetics and environment on isoflavone content of soybean from different regions of Brazil. Pesquisa Agropecuária Brasileira, Brasília, v. 34, n. 10, p. 1787-1795, out. 1999.

CARRÃO-PANIZZI, M. C.; KITAMURA, K. Isoflavone content in Brazilian soybean cultivars. Breeding Science, Tokyo, v. 45, n. 3, p. 295-300, 1995.

CARRÃO-PANIZZI, M. C.; KITAMURA, K.; BELÉIA, A. D. P.; OLIVEIRA, M. C. N. Influence of growth locations on isoflavone contents in Brazilian soybean cultivars. Breeding Science, Tokyo, v. 48, p. 409-413, 1998.

COCHRAN, W. G.; COX, G. M. Experimental designs. New York: J. Willey, 1957. 611 p.

COWARD, L.; BARNES, N. C.; SETCHELL, K. D. R.; BARNES, S. Genistein, daidzein, and their $\beta$-glycoside conjugates: antitumor isoflavones in soybean foods from American and Asian diets. Journal of Agricultural and Food Chemistry, Washington, v. 41, p. 1961-1967, 1993.

COWARD, L.; SMITH, M.; KIRK, M.; BARNES, S. Chemical modification of isoflavones in soyfoods during cooking and processing. American Journal of Clinical Nutrition, Bethesda, v. 68, n. 6, p. 1486S-1491S, 1998. Supplement. 
ELDRIDGE, A.; KWOLEK, W. Soybean isoflavones: effect of environment and variety on composition. Journal of Agricultural and Food Chemistry, Washington, v. 31, n. 2, p. 394-396, 1983.

HARTLEY, H. O. Testing the homogeneity of a set of variances. Biometrika, Oxford, v. 31, p. 249-255, 1940.

KITAMURA, K.; IGITA, K.; KIKUCHI, K.; KUDOU, $\mathrm{S}$.; OKUBO, K. Low isoflavone content in early maturing cultivars, so called summer-type soybeans (Glycine max (L.) Merrill). Japanese Journal of Breeding, Tokyo, v. 41, p. 651-654, 1991

KUDOU, S.; FLEURY, Y.; WELTI, D.; MAGNOLATO, D.; UCHIDA, T.; KITAMURA, K.; OKUBO, K. Malonyl isoflavone glycosides in soybeans seeds (Glycine max Merrill). Agricultural and Biological Chemistry, Tokyo, v. 55, p. 2227-2233, 1991.

MATSUURA, M.; OBATA, A. $\beta$-glucosidases from soybean hydrolyse daidzin and genistin. Journal of Food Science, Chicago, v. 58, n. 1, p. 144-147, 1993.

MATSUURA, M.; OBATA, A.; FUKUSHIMA, D. Objectionable flavor of soymilk developed during the soaking of soybeans and its control. Journal of Food Science, Chicago, v. 54, p. 602-605, 1989.

PARK, Y. K.; AGUIAR, C. L.; ALENCAR, S. M.; SCAMPARINI, A. R. P. Biotransformação de 2-glicosil isoflavonas de soja em isoflavonas agliconas por 2-glicosidase fúngica. In: SIMPÓSIO BRASILEIRO SOBRE OS BENEFÍCIOS DA SOJA PARA A SAÚDE HUMANA, 1., 2001, Londrina. Anais... Londrina: Embrapa-CNPSo, 2001. p. 33-36. (Documentos, 169).
PETERSON, G.; BARNES, S. Genistein and biochanin A inhibit the growth of human prostate cancer cells but not epidermal growth factor receptor tyrosine autophosphorylation. Prostate, New York, v. 22, n. 4, p. $335-345,1993$.

SAS INSTITUTE (Cary, Estados Unidos). SAS/STAT software: changes and enhancements through release 6.12. Cary, 1997. $1167 \mathrm{p}$.

SETCHELL, K. D. R.; BROWN, N. M.; DESAI, P.; ZIMMER-NECHEMIAS, L.; WOLFE, B. E.; BRASHEAR, W. T.; KIRSCHNER, A. S.; CASSIDY, A.; HEUBI, J. E. Bioavailability of pure isoflavones in healthy humans and analysis of commercial soy isoflavone supplements. Journal of Nutrition, Bethesda, v. 131, p. 1362S-1375S, 2001.

SHAPIRO, S. S.; WILK, M. B. An analysis of variance test for normality. Biometrika, Oxford, v. 52, p. 591-611, 1965.

TAM, D. M.; GARDNER, C. D.; HASKELL, W. L. Potential health benefits of dietary phytoestrogens: a review of the clinical, epidemiological and mechanistic evidence. Journal of Clinical Endocrinology and Metabolism, Bethesda, v. 83, p. 2223-2235, 1998.

TSUKAMOTO, C.; SHIMADA, S.; IGITA, K.; KUDOU, S.; KOKUBUN, M.; OKUBO, K.; KITAMURA, K. Factors affecting isoflavones content in soybean seeds: changes in isoflavones, saponins and composition of fatty acids at different temperatures during seed development. Journal of Agriculture and Food Chemistry, Washington, v. 43, p. 1184-1192, 1995.

TUKEY, J. W. One degree of freedom for non-additivity. Biometrics, Washington, v. 5, p. 232-242, 1949. 